\title{
Neural Network Classifier for Hand Motion Detection from EMG Signal
}

\author{
Md. R. Ahsan, M.I. Ibrahimy, and O.O. Khalifa \\ Department of Electrical \& Computer Engineering, Kulliyyah of Engineering, \\ International Islamic University Malaysia, Kuala Lumpur
}

\begin{abstract}
EMG signal based research is ongoing for the development of simple, robust, user friendly, efficient interfacing devices/systems for the disabled. The advancement can be observed in the area of robotic devices, prosthesis limb, exoskeleton, wearable computer, $\mathrm{I} / \mathrm{O}$ for virtual reality games and physical exercise equipments. Additionally, electromyography (EMG) signals can also be applied in the field of human computer interaction (HCI) system. This paper represents the detection of different predefined hand motions (left, right, up and down) using artificial neural network (ANN). A backpropagation (BP) network with Levenberg-Marquardt training algorithm has been utilized for the classification of EMG signals. The conventional and most effective time and timefrequency based feature set is utilized for the training of neural network. The obtained results show that the designed network is able to recognize hand movements with satisfied classification efficiency in average of $\mathbf{8 8 . 4 \%}$. Furthermore, when the trained network tested on unknown data set, it successfully identify the movement types.
\end{abstract}

Keywords - Electromyography, Human Computer Interaction, Artificial Neural Network, Discrete Wavelet Transform.

\section{INTRODUCTION}

In the past three decades, the development of EMG based control has got the focus in the sense that it will increase the social acceptance of the disabled and aged people by improving their quality of life. In fact, EMG based input device is a natural means of HCI because the electrical activity induced by the human's arm muscle movements can be interpreted and transformed into computer's control commands. However, the most difficult part for developing myoelectric control based interfaces is the pattern recognition of EMG signals. This is because of large variations in EMG signals having different signatures depending on age, muscles activity, motor unit paths, skin-fat layer, and gesture style. Compared to other biosignals, EMG signal contains complicated types of noise that are caused by inherent equipment and environment noise, electromagnetic radiation, motion artifacts, and the interaction of different tissues. Sometimes it is difficult to extract useful features from the residual muscles of an amputee or disabled. This difficulty becomes more critical when it is resolving a multiclass classifying problem [1].
For the purpose of classification, the EMG pattern signatures are extracted for each movement and then a proper discrimination method is applied to classify the EMG signals based on features. However, it is difficult to have a precise structural or mathematical model of EMG signals that relates the measured signals to motion command. There are many pattern recognition techniques available to discriminate the functionality from extracted features. It has been found that most of researchers used ANN for the processing of biosignals [2]. ANNs are particularly useful for complex pattern recognition and classification tasks. The capability of learning from examples, the ability to reproduce arbitrary non-linear functions of input, and the highly parallel and regular structure of ANNs make them especially suitable for pattern classification tasks [3]. In this regard, integral absolute value (IAV) feature based feed-forward ANN used by Hiraiwa et. al [4], independent component analysis (ICA) based ANN by Naik et. al. [5], different multi-layer perceptron (MLP) based neural network used by Englehart et. al.[6], Kelly et. al. [7], prominent researchers Hudgins et al. have used Hopfield and ART, and later finite impulse response neural network (FIRNN) [8]. Most of the ANN based research work has been carried out with MLP containing one hidden layer and back-propagation (BP) algorithm for training. Varying accuracy in all the above neural network based classification techniques may be due to quality of collected signal, signal conditioning along with physiological characteristics of the subject.

This paper describes the classification of EMG signals by using ANN. Different features were calculated for EMG signals and applied to Levenberge-Marquardt algorithm based neural network for hand movement realization. Acceptable success rate of classification has been achieved and reported in result and discussion section.

\section{Methodology}

Signal Acquisition: The device used for the acquisition of EMG signals was Biopac MP100 data acquisition system (EMG-100C amplifier) along with the acquisition software AcqKnowledge 3.9.1. The EMG signals were collected from three able-bodied persons age between 27-32. The sampling frequency was $1000 \mathrm{~Hz}$ and gain for the acquired 
signal was 1000. The optimal position of the electrode placement is determined by performing several trials of the acquisition experiment. The single channel differential electrodes placed on brachioradialis \& flexor carpum ulnaris muscle and the reference electrode on the wrist. The subjects were requested to perform some predefined voluntary movements of hand in different direction (Left, Right, Up, Down) and then the EMG signals collected from the muscle. It was found that the average time required to perform each movements was around 500 miliseconds. Each EMG Signal set has been collected for 70 seconds including 5 seconds rest in start and end of the signal acquisition. The EMG signals stored in a Windows XP based personal computer (PC) for post analysis and processing.

Preprocessing of EMG Signal: Because of very sensitive nature of EMG signals, it can be easily contaminated by different kind of noise sources and artifacts which will contribute in very poor classification result. The noise can be eliminated by using typical filtering procedures such as band-pass filter, band-stop filter or the use of a good quality of equipment with a proper electrode placement. Whereas it is difficult to remove the effect of other noises/artifacts and interferences of random noise that is in between dominant frequency range of EMG [9]. A six order Butterworth bandpass filter with cut-off $20-500 \mathrm{~Hz}$ used to eliminate the first three types of noise. The $50 \mathrm{~Hz}$ power line noise was removed by notch filter with $3 \mathrm{db}$ gain and $49-51 \mathrm{~Hz}$ since it was found the dominant frequency in the range of 70-300 Hz. The frequency domain presentation of filter input and output is shown in Fig. 1. Afterwards, the EMG signals were denoised using wavelet method. Wavelet techniques can successfully localize both time and frequency components and signals are processed in various scales/resolutions. Moreover, wavelet processing provides good frequency resolution at high frequencies. As a result, the noise components in the desired signal can be easily isolated by preserving important high-frequency transients [10]. EMG signal for 4 different hand movements (left, right, up, down) was segmented and each segment consists of 500 data points. For the discrete wavelet transform (DWT), four level decompositions of EMG signal were used. Daubechies (db2) mother wavelet function was selected and applied on detail wavelet coefficients for noise reduction. Later on, the features were extracted for each type of hand movement from the denoised EMG signals.

Feature Extraction: For efficient classification of EMG signals, it is important to select proper features. The success of pattern classification system depends completely on the choice of features used to represent the raw EMG signals [8]. It is necessary to use multiple feature parameters for EMG signal classification since it is quite difficult to extract a feature parameter which reflects the unique feature of the measured EMG signals to a motion command perfectly. It has been found that many researchers have selected time domain, frequency domain, time-frequency domain and time-scale domain features for the classification of EMG signals. Various type of features extracted by different researchers such as mean absolute value (MAV), root mean square (RMS), auto-regression (AR) coefficients, variance (VAR), standard deviation (SD), zero crossing (ZC), waveform length (WL), Willson amplitude (WA), mean absolute value slope (MAVS), mean frequency (MNF), median frequency (MDF)slope sign change (SSC), cepstrum coefficients (CC), fast Fourier transform (FFT) coefficients, short time Fourier transform (STFT) coefficients, integrated EMG (IEMG), wavelet transform (WT) coefficients, and wavelet packet transform (WPT) coefficients [8], [11], [12]. In this work, seven statistical time and time-frequency based features namely MAV, RMS, VAR, SD, ZC, SSC and WL are used.

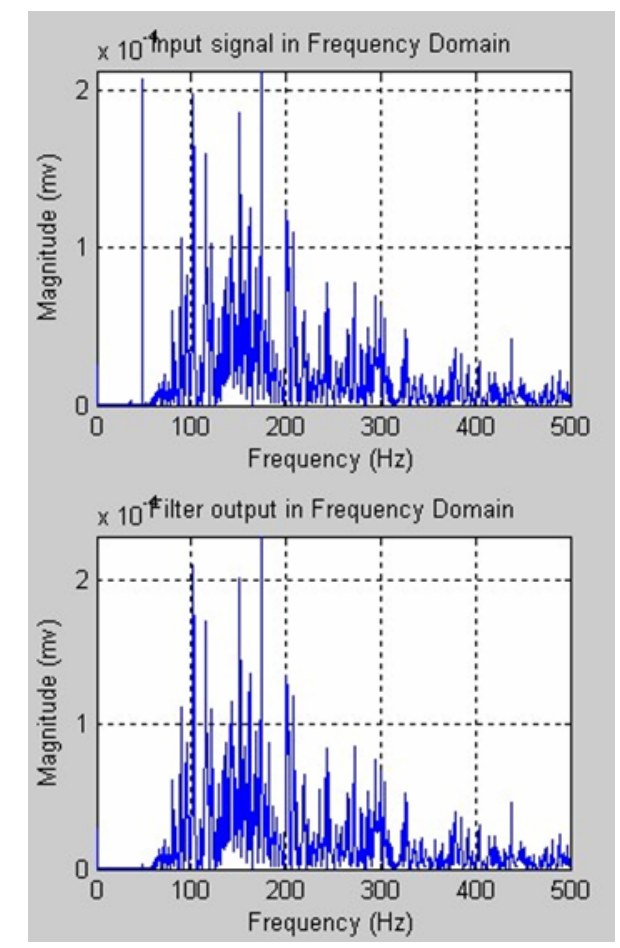

Fig. 1 Removing of $50 \mathrm{~Hz}$ electrical noise

Neural Network Architecture for EMG Classification: BP is based on the generalized form of Widrow-Hoff learning rule to multiple-layer network and nonlinear differentiable transfer function. Here, the input vectors and corresponding target vectors are used to train the neural network until it can approximate a function or associate input vectors with 
specific output vectors or classify input vectors in an appropriate way based on certain criteria. The designed network consists of three-layers: input layer, tan-sigmoid hidden layer and linear output layer. Each layer except input layer has a weight matrix $\mathrm{W}$, a bias vector $\mathrm{b}$ and an output vector a. The weight matrices connected to inputs called input weights (IW) and weight matrices coming from hidden layer outputs called layer weights (LW). Additionally, superscripts are used to denote the source (second index) and the destination (first index) for the various weights and other elements of the network.

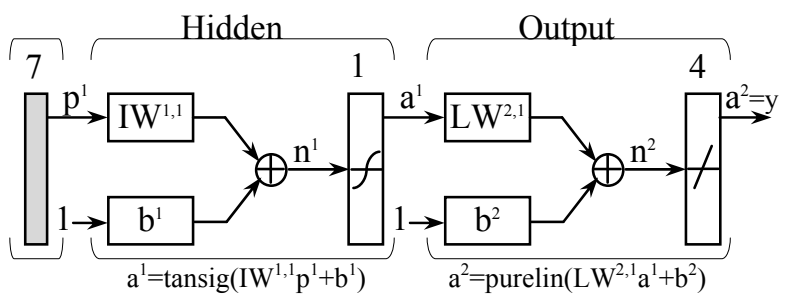

Fig. 2 Architecture of Artificial Neural Network

The feedforward BP network architecture has shown in Fig. 2 with seven neurons in input layer, 10 tan-sigmoid neurons in hidden layer and four linear neurons in output layer. Since there is no specific way to find out the number of hidden neurons, so it has been determined from best classification result by selecting different numbers of neurons. The predefined features were extracted for four types of hand movements from five different EMG signals. 204 sets of input feature vectors from four EMG signals and their corresponding target vectors were fed to the network for training purpose. The feature vectors from remaining EMG signal used for testing the performance of network. The input feature vectors were normalized before feeding for the purpose of efficient training of neural network. The sample input vectors and corresponding target vectors for four movements are shown in Table.1.

Levenberg-Marquardt (trainlm) algorithm was utilized for BP training. It is the fastest method for training of moderate-sized feedforward neural networks and based on numerical optimization techniques. The network was also generalized to avoid overfitting. This was done by dividing the training input data: $70 \%$ for training, $15 \%$ for validation and $15 \%$ for testing. Furthermore, the number of data points in training set was more than sufficient to estimate the total number of parameters in the network. Two early stopping conditions were used to improve the generalization of network. The training of the network will stop if total mean squared error $<=0.001$ or when it reaches to 1000 epochs. The weights and bias of input layer and hidden layer were saved after each training session. When the simulation results are not satisfactory, the network trained again with the last saved weight and bias values. This was done to improve the network performance and to reduce the number of time for training. Another type of BP algorithm named scaled conjugate gradient (trainscg) is also used and reported in result section.

Table 1 Sample feature sets for different movement with target vectors of corresponding movement

\begin{tabular}{|c|c|c|c|c|c|}
\hline \multirow{9}{*}{ 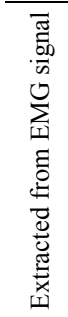 } & \multirow{2}{*}{ Feature } & \multicolumn{4}{|c|}{ Movement type } \\
\hline & & Left & Right & $\mathrm{Up}$ & Down \\
\hline & MAV & 0.11862 & 0.10395 & 0.10554 & 0.07303 \\
\hline & RMS & 0.16918 & 0.14866 & 0.14803 & 0.09834 \\
\hline & VAR & 0.02862 & 0.02210 & 0.02191 & 0.00967 \\
\hline & $\mathrm{SD}$ & 0.16910 & 0.14861 & 0.14811 & 0.09841 \\
\hline & WL & 72.66327 & 65.94147 & 62.51984 & 45.28778 \\
\hline & SSC & 234 & 214 & 220 & 230 \\
\hline & $\mathrm{ZC}$ & 207 & 204 & 191 & 219 \\
\hline \multirow{13}{*}{ 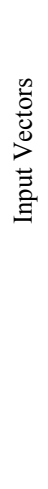 } & \multicolumn{5}{|c|}{ After normalization in the range $\left[\begin{array}{ll}-1 & 1\end{array}\right]$} \\
\hline & MAV & 0.62547 & 0.36995 & 0.39767 & -0.16858 \\
\hline & RMS & 0.57261 & 0.33743 & 0.33016 & -0.23938 \\
\hline & VAR & 0.32669 & 0.01320 & 0.00415 & -0.58431 \\
\hline & $\mathrm{SD}$ & 0.57222 & 0.33665 & 0.33096 & -0.24050 \\
\hline & WL & 0.55323 & 0.37111 & 0.27841 & -0.18846 \\
\hline & SSC & 0.10811 & -0.43243 & -0.27027 & 0.00000 \\
\hline & $\mathrm{ZC}$ & -0.03226 & -0.12903 & -0.54839 & 0.35484 \\
\hline & & \multicolumn{4}{|c|}{ Target Vector } \\
\hline & & 1 & 0 & 0 & 0 \\
\hline & & 0 & 1 & 0 & 0 \\
\hline & & 0 & 0 & 1 & 0 \\
\hline & & 0 & 0 & 0 & 1 \\
\hline
\end{tabular}

\section{Results AND DISCUSSION}

The summery of the classification performance using back-propagation neural network is shown in Table 2. The network was trained by 204 sets of data for different movements. Each set consists of input feature vector obtained from specific type of hand movement and corresponding output vector. Different number of hidden neurons selected for both type of BP training and their classification efficiency are reported. It is found that Levenberg-Marquardt algorithm based back-propagation neural network with 10 hidden neurons yields the best classification rate and required processing time is less. This network outperforms others regarding number of iterations required, time elapsed and classification rate. The best validation performance achieved at 10 epochs and the training stopped at 16 epochs as shown in Fig. 3. The number presentations of the classes are: 1 for left, 2 for right, 3 for up and 4 for down. The average of best overall classification rate during training is found $88.4 \%$. The detailed performances of network during 
training, validation, testing and overall during a single trial are shown by confusion matrix for different class in Fig. 4. The trained network has also been tested on completely unknown EMG signals. The feature vectors are fed to the network without the corresponding targets. The expected output was 1 in its index position for a specific type of movement. The sample input feature vectors from test EMG signal and its corresponding output are shown in Table 3. The classified movements are presented in bold numbers which are the largest others and closer to 1 . The test output shows that the trained network successfully classified all the movement types.
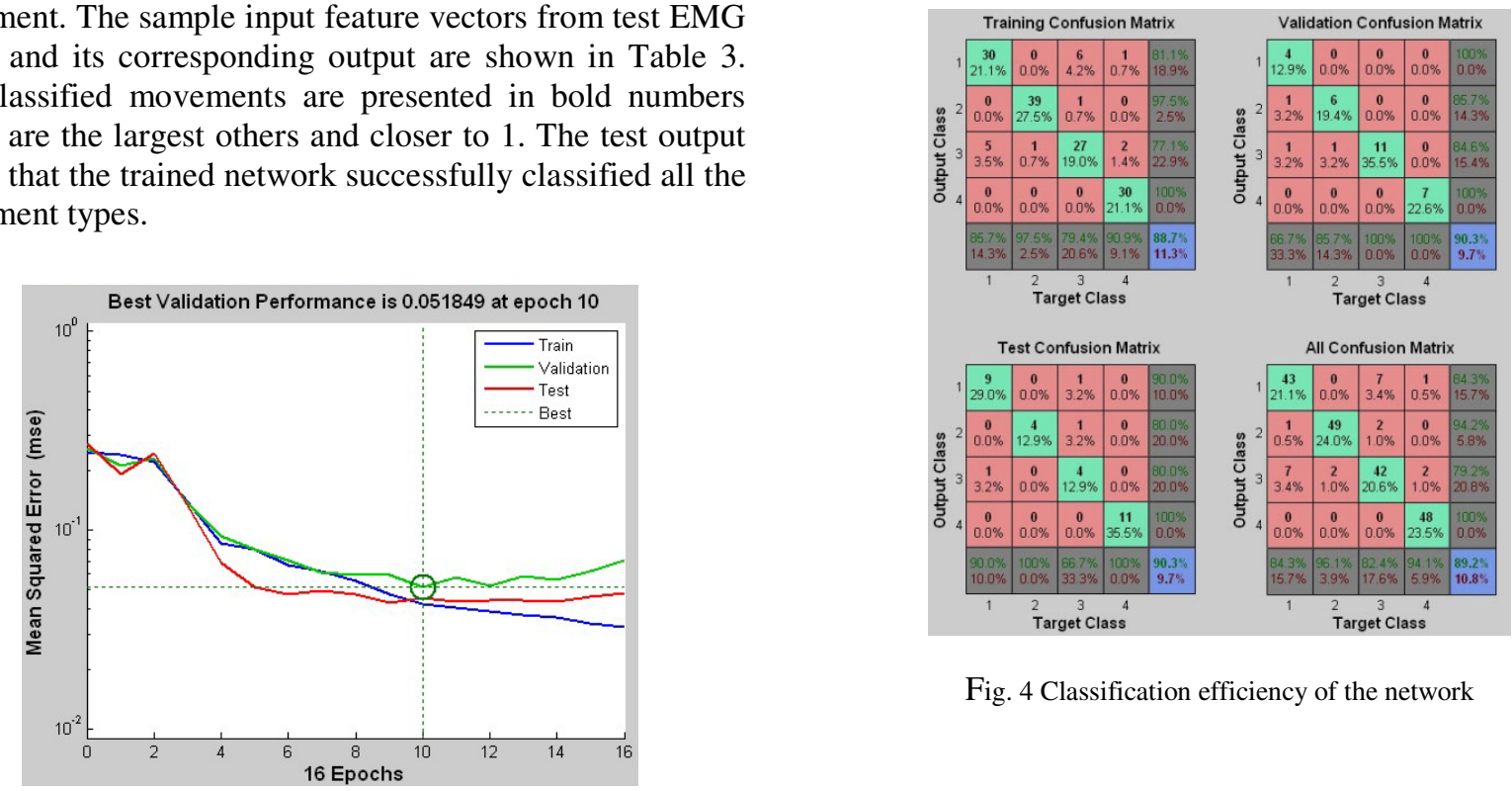

Fig. 4 Classification efficiency of the network

Fig. 3 The early stopping criteria

Table 2 Experimental result with comparison

\begin{tabular}{|c|c|c|c|c|c|c|c|c|}
\hline \multirow{2}{*}{$\begin{array}{l}\text { Training } \\
\text { Function }\end{array}$} & \multirow{2}{*}{$\begin{array}{l}\text { Stop Ep- } \\
\text { ochs }\end{array}$} & \multirow{2}{*}{$\begin{array}{l}\text { Regre- } \\
\text { ssion }\end{array}$} & \multirow{2}{*}{$\begin{array}{c}\text { Time } \\
\text { Elapsed }\end{array}$} & \multicolumn{4}{|c|}{ Classification Rate } & \multirow{2}{*}{$\begin{array}{c}\text { Hidden } \\
\text { Neurons }\end{array}$} \\
\hline & & & & Training & Validation & Test & Overall & \\
\hline \multirow{12}{*}{ 志 } & 15 & 0.8597 & 1.047 & 88.6 & 83.3 & 90 & 88 & \multirow{4}{*}{10} \\
\hline & 18 & 0.87251 & 0.921 & 94.3 & 66.7 & 80 & 88 & \\
\hline & 16 & 0.87401 & 0.8721 & 88.7 & 90.3 & 90.3 & 89.2 & \\
\hline & Avg & 0.86874 & 0.9467 & 90.5333 & 80.1 & 86.76667 & 88.4 & \\
\hline & 33 & 0.85706 & 2.797 & 91.4 & 70 & 83.3 & 87 & \multirow{4}{*}{20} \\
\hline & 14 & 0.85508 & 1.218 & 90 & 80 & 86.7 & 88 & \\
\hline & 12 & 0.84772 & 1.094 & 92.9 & 76.7 & 83.3 & 89 & \\
\hline & Avg & 0.853287 & 1.703 & 91.4333 & 75.5667 & 84.43333 & 88 & \\
\hline & 16 & 0.86112 & 2.36 & 92.1 & 80 & 76.7 & 88 & \multirow{4}{*}{30} \\
\hline & 11 & 0.85018 & 1.703 & 91.4 & 90 & 73.3 & 88.5 & \\
\hline & 14 & 0.85102 & 2.125 & 89.3 & 76.7 & 83.3 & 86.5 & \\
\hline & Avg & 0.854107 & 2.06267 & 90.9333 & 82.2333 & 77.76667 & 87.6667 & \\
\hline \multirow{4}{*}{ 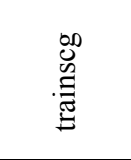 } & 37 & 0.7839 & 0.703 & 80.7 & 83.3 & 83.3 & 81.5 & 10 \\
\hline & 31 & 0.80202 & 0.797 & 78.6 & 90 & 86.7 & 81.5 & 20 \\
\hline & 34 & 0.80767 & 0.859 & 83.6 & 83.3 & 86.7 & 84 & 30 \\
\hline & Avg & 0.797863 & 0.78633 & 80.96667 & 85.5333 & 85.56667 & 82.3333 & \\
\hline
\end{tabular}


Table 3 Sample test data with classification output

\begin{tabular}{|c|c|c|c|c|c|c|c|c|c|c|}
\hline Input> & $\mathrm{p} 1$ & $\mathrm{p} 2$ & p3 & p4 & p5 & p6 & p7 & $\mathrm{p} 8$ & p9 & p10 \\
\hline \multirow{7}{*}{ 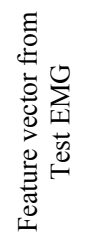 } & -0.12863 & -0.61608 & 0.37114 & 0.20342 & -0.13348 & -0.75516 & 0.22405 & 0.33569 & -0.24777 & -0.79584 \\
\hline & -0.12208 & -0.66515 & 0.34085 & 0.16422 & -0.22866 & -0.77763 & 0.09469 & 0.32470 & -0.34035 & -0.81355 \\
\hline & -0.51192 & -0.88580 & -0.00891 & -0.22084 & -0.60371 & -0.93406 & -0.29750 & -0.02931 & -0.69024 & -0.94736 \\
\hline & -0.12391 & -0.66707 & 0.33959 & 0.16281 & -0.23027 & -0.77885 & 0.09339 & 0.32338 & -0.34266 & -0.81470 \\
\hline & -0.17617 & -0.64118 & 0.19024 & 0.32336 & -0.13286 & -0.75488 & 0.09132 & 0.46075 & -0.34527 & -0.78546 \\
\hline & -0.29730 & 0.40541 & 0.10811 & -0.78378 & -0.05405 & 0.45946 & 0.29730 & -0.56757 & 0.08108 & 0.00000 \\
\hline & -0.42105 & -0.55263 & -0.65789 & 0.31579 & -0.23684 & 0.02632 & -0.52632 & 0.50000 & -0.55263 & -0.50000 \\
\hline \multirow{4}{*}{ 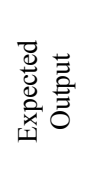 } & 0 & 0 & 1 & 0 & 0 & 0 & 1 & 0 & 0 & 0 \\
\hline & 0 & 0 & 0 & 1 & 0 & 0 & 0 & 1 & 0 & 0 \\
\hline & 1 & 0 & 0 & 0 & 1 & 0 & 0 & 0 & 1 & 0 \\
\hline & 0 & 1 & 0 & 0 & 0 & 1 & 0 & 0 & 0 & 1 \\
\hline \multirow{4}{*}{ 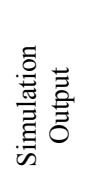 } & 0.0036 & 0.0004 & 0.6597 & 0.0081 & 0.0447 & 0.0024 & 0.6676 & 0.0273 & 0.0063 & 0 \\
\hline & 0.0085 & 0.0036 & 0.0032 & 0.955 & 0.0021 & 0 & 0.0017 & 0.8774 & 0.0003 & 0.0133 \\
\hline & 0.9928 & 0.0001 & 0.3271 & 0.091 & 0.7688 & 0.0018 & 0.5993 & 0.1906 & 0.8913 & 0.0008 \\
\hline & 0.0548 & 0.9991 & 0.02 & 0 & 0.1496 & 0.998 & 0.0093 & 0 & 0.288 & 0.9995 \\
\hline
\end{tabular}

\section{Conclusions}

The research work has been carried out for the classification of different hand movements based on EMG signals. The classified signal can be utilized for the controlling of any human-computer centered systems or devices. The experimental result shows that the Levenberg-Marquardt algorithm based neural network recognizes the desired motions efficiently and takes minimal computation time. It has been found that the designed ANN has successfully classified the EMG signals from hand movements and the average success rate is $88.4 \%$. Whereas in a single trial the best overall performance has been found $89.2 \%$. The performance obtained without any prior training of the subject's hand movements. It can be conclude that the classification efficiency will increase if the network supplied with rich EMG signals. This can be done either by short training of the subject which can reproduce repeatable signals or by allowing the network to adapt with changes of feature value. In that situation, a good classification can be obtained and which will lead to the development of suitable HCI system. The classified EMG signal can be efficiently used to develop a human computer interface to help the people with disabilities who wish to interact with computer devices.

\section{REFERENCES}

1. Kim, J, Mastnik, S André, E. EMG-based hand gesture recognition for realtime biosignal interfacing. Proc ACM IUI '08, 30 39:

2. Ahsan, M, Ibrahimy, M Khalifa, O. Advances in electromyogram signal classification to improve the quality of life for the disabled and aged people. Journal of Computer Science 2010; 6:706-715

3. Subasi, A, Yilmaz, M Ozcalik, HR. Classification of EMG signals using wavelet neural network. Journal of neuroscience methods 2006; 156:360-367

4. Hiraiwa, A, Shimohara, K Tokunaga, Y. EMG pattern analysis and classification by neural network. Systems, Man and Cybernetics, 1989. Conference Proceedings., IEEE International Conference on 2002; 1113-1115

5. Naik, GR, Kumar, DK, Singh, VP et al. Hand gestures for HCI using ICA of EMG. Proceedings of the HCSNet workshop on Use of vision in human-computer interaction-Volume 56 2006; 67-72

6. Englehart, K, Hudgins, B, Stevenson, M et al. A dynamic feedforward neural network for subset classification of myoelectric signal patterns. Engineering in Medicine and Biology Society, 1995., IEEE 17th Annual Conference 2002; 1:819-820

7. Kelly, MF, Parker, PA Scott, RN. The application of neural networks to myoelectric signal analysis: a preliminary study. Biomedical Engineering, IEEE Transactions on 2002; 37:221-230

8. Hudgins, B, Parker, P Scott, R. A new strategy for multifunction myoelectric control. Biomedical Engineering, IEEE Transactions on 1993; 40:82-94

9. Reaz, MB, Hussain, MS Mohd-Yasin, F. Techniques of EMG signal analysis: detection, processing, classification and applications. Biological procedures online 2006; 8:11-35 
10. Hussain, MS, Reaz, MBI Ibrahimy, MI. SEMG signal processing and analysis using wavelet transform and higher order statistics to characterize muscle force. Proceedings of the 12th WSEAS international conference on Systems 2008; 366-371

11. Phinyomark, A, Limsakul, C Phukpattaranont, P. A novel feature extraction for robust EMG pattern recognition. Journal of Computing 2009; 1:71-80

12. Tsenov, G, Zeghbib, A, Palis, F et al. Neural Networks for Online Classification of Hand and Finger Movements Using Surface EMG signals. Neural Network Applications in Electrical Engineering, 2006. NEUREL 2006. 8th Seminar on 2006; 167-171
The address of the corresponding author:

Author: Md. Rezwanul Ahsan

Institute: International Islamic University Malaysia

Street: Jalan Gombak

City: Kuala Lumpur

Country: Malaysia

Email: rezwanul.ahsan@yahoo.com 